\title{
METHOXYFLURANE ET NEPHROTOXICITE: ETUDE DE LA FONCTION RENALE DE 22 MALADES ANESTHESIES AU METHOXYFLURANE*
}

GÉrard Mignault, M.D., Benoît LabrecQue, M.D., F.R.C.P.(c), ET SUZANne hamel $†$

LE MÉTHOXYFLURANe est sûrement l'un des agents anesthésiques précieux que nous possédions présentement. Ses propriétés analgésiques marquées sont particulièrement favorables à l'obstétrique, à la chirurgie digestive haute (gastrectomie, vagotomie). Les malades souffrant d'atteinte hépatique bénéficient de sa faible toxicité hépatique, de même que ceux qui ont subi des anesthésies longues ou multiples récentes à l'halothane. Son association possible à l'adrénaline est précieuse au cours de la chirurgie plastique ou oto-rhino-laryngologique.

Cependant, certains travaux récents ${ }^{1,2}$ rapportent un effet néphrotoxique du composé se manifestant cliniquement par une insuffisance rénale à diurèse élevée: "high output renal failure." Le rein serait incapable de retenir l'eau, le débit urinaire augmentant sans proportion avec l'hydratation, fut-elle déficiente. Cette diurèse excessive peut conduire à une déshydratation, une perte de poids marquée, une élévation de l'urée sanguine et une hypernatrémie.

Crandell ${ }^{2}$ mentionne une incidence d'atteinte rénale de 17 pour cent de ses malades soumis à l’administration de méthoxyflurane. Il nous a semblé intéressant de vérifier l'incidence et le type d'atteinte rénale dans notre contexte d'utilisation de cet agent.

\section{MÉTHODES}

Choix des malades (Tableau I)

Vingt-deux malades devant subir une intervention chirurgicale majeure ont été choisis au hazard et anesthésiés au méthoxyflurane (Tableau I). Ce groupe comprend 6 femmes et 16 hommes. La nature des interventions se répartit comme suit: chirurgie vasculaire, 5 cas; chirurgie digestive, 6 cas; chirurgie orthopédique, 8 cas; chirurgie gynécologique, 1 cas; chirurgie thoracique, 1 cas; chirurgie plastique, 1 cas.

\section{Evaluation préopératoire}

Aucun de ces malades ne présentait d'anomalie au bilan biologique habituel établi en préopératoire. Ceci comprend, en particulier, l'hémogramme, l'analyse d'urine, l'urée sanguine, la créatinine, le sodium, le potassium plasmatique.

- Travail présenté à la session d'automne de la Société canadienne des anesthésistes, division du Québec.

tLes docteurs Mignault et Labrecque sont membres du Service d'anesthésie de l'Hôtel-Dieu de Montréal. Mlle Hamel est stagiaire d'été, étudiante en médecine à l'Université de Montréal.

Canad. Anaesth. Soc. J., vol. 17, no. 4, July 1970 
TABLEAU I

SuJETS DE L'ÉTUdE

\begin{tabular}{|c|c|c|c|c|}
\hline Dossier & Nom & Age (ans.) & Operation & $\begin{array}{c}\text { Durée anesthésie } \\
\text { (hres.) }\end{array}$ \\
\hline 69-84-682 & M.E.F. & 57 & Greffe aorto-fémoro & 1.00 \\
\hline $69-72-205$ & Mme A.L. & 48 & $\begin{array}{l}\text { Lophération d'adhérences, } \\
\text { pyloroplastie, vagotomie bil. }\end{array}$ & 3.30 \\
\hline $69-109-898$ & M. J.H. & 61 & $\begin{array}{l}\text { Thoracostomie gauche, } \\
\text { biopsie, décortication pulm. }\end{array}$ & 5.45 \\
\hline $69-101-090$ & Mme A.L. & 39 & Wertheim & 4.00 \\
\hline $69-110-037^{*}$ & M. D.T. & 50 & $\begin{array}{l}\text { Arthroplastie hanche gauche } \\
\text { (cupule) }\end{array}$ & 4.40 \\
\hline $69-110-073$ & M. H.C. & 56 & $\begin{array}{l}\text { Sympathectomie lomb. bil. \& } \\
\text { greffe fémoro popl. droite }\end{array}$ & 4.30 \\
\hline $\begin{array}{l}69-110-161 \\
69-110-242 \\
69-100-768 \\
69-110-290 \\
69-110-489 \\
69-110-654\end{array}$ & $\begin{array}{l}\text { M. J.P.C. } \\
\text { M. E.C. } \\
\text { M. G.L. } \\
\text { M. G.M. } \\
\text { Mme M.T. } \\
\text { M. L.L. }\end{array}$ & $\begin{array}{l}44 \\
69 \\
49 \\
55 \\
57 \\
55\end{array}$ & $\begin{array}{l}\text { Discoidectomie cerv., Greffe } \\
\text { Arthrotomie épaule droite } \\
\text { Cholécystectonie } \\
\text { Sympathectomie lomb. bil. } \\
\text { Arthrotomie hanche droite } \\
\text { Arthrodèse gros orteil, } \\
\text { ostéotomie tibiale, cure } \\
\text { d'hydrocèle \& exer. kyste }\end{array}$ & $\begin{array}{l}3.00 \\
1.35 \\
2.30 \\
1.50 \\
6.00 \\
1.45\end{array}$ \\
\hline $69-94-879$ & M. R.P. & 45 & Discoidectomie L4 à gauche & 2.00 \\
\hline $69-110-634$ & Mme R.D.R. & 71 & Enclouage hanche gauche & 2.50 \\
\hline $69-110-761$ & M. R.M. & 68 & Dérivation fémoro popl. droite & 4.10 \\
\hline $69-110-749$ & Mlle J.D. & 15 & Cure hernie ing. bil. & 2.15 \\
\hline $69-110-847$ & M. A.L. & 64 & $\begin{array}{l}\text { Réduction fract. cheville et } \\
\text { plâtre }\end{array}$ & 0.20 \\
\hline $69-110-831$ & M. P.S. & 18 & Arthrotomie épaule droite & 2.15 \\
\hline $69-99-360$ & M. E.F. & 60 & Sympathectomie lomb. gauche & 2.10 \\
\hline $69-110-947$ & Mme F.S. & 60 & Gastrectomie, vagotomie bil. & 3.30 \\
\hline $69-64-506$ & M. G.D. & 69 & $\begin{array}{l}\text { Lambeau cuir chevelu et } \\
\text { exer. total os temp. droit et } \\
\text { exer, oreilles int. \& moy. }\end{array}$ & 15.00 \\
\hline $69-110-760$ & Frère R.H. & 56 & $\begin{array}{l}\text { Cholécystectomie et cure } \\
\text { hernie ing. droite et } \\
\text { appendicectomie }\end{array}$ & 2.50 \\
\hline
\end{tabular}

*Cas positif de l'étude.

\section{Technique d'anesthésie}

Nous avons pratiqué une anesthésie balancée où chaque agent est employé pour un effet pharmacologique spécifique, le méthoxyflurane étant employé uniquement pour ses propriétés analgésiques. L'induction a été faite par injection d'une dose hypnotique de thiopental ( 1 à $2 \mathrm{mg} / \mathrm{lb}$ de poids corporel) et de succinylcholine $(0.4$ à $0.6 \mathrm{mg} / \mathrm{lb}$ de poids corporel) suivie d'une hyperventilation manuelle avec mélange $\mathrm{N}_{2} \mathrm{O}+\mathrm{O}_{2} 50 / 50$ ou $\mathrm{O}_{2}$ à 100 pour cent; l'intubation orotrachéale a été pratiquée sous vision directe puis, hyperventilation modérée, assurée par respirateur, avec mélange $\mathrm{N}_{2} \mathrm{O}+\mathrm{O}_{2} 60 / 40$ ou 50/50; la curarisation fut maintenue par la suite par administration de d-tubocurarine $(0.25$ à $1 \mathrm{mg} / 4 \mathrm{lb}$ de poids corporel) selon les besoins de la chirurgie en cours. Le méthoxyflurane a été vaporisé par "Pentec" et ajouté au mélange inhalatoire peu après l'initiation de la ventilation artificielle, aux concentrations de 0.5 parfois 1 pour cent pour l'induction, puis 0.2 à 0.5 pour cent pour le maintien, selon les signes cliniques de l'anesthésie, la tension artérielle, etc. A l'occasion, l'analgésie a été 
complétée par l'injection intraveineuse de mépéridine à raison de $10 \mathrm{mg}$ par injection.

Paramètres de la fonction rénale proprement dite

En ce qui concerne plus spécifiquement la fonction rénale, les paramètres suivants ont été mesurés quotidiennement à partir du jour préopératoire (jour 0 ) jusqu'au $4^{\mathrm{e}}$ jour postopératoire inclusivement: ingesta et excreta, osmolalité urinaire; urée sanguine, créatinine et sodium plasmatiques; une épreuve à la vasopressine (voir section "résultats") était prévue pour le $5^{\mathrm{e}}$ jour postopératoire advenant l'apparition de signes d'altération de la fonction rénale.

\section{RÉSULTats}

Débit urinaire (Fig. 1, Tableau II)

Les valeurs de la diurèse horaire des sujets de l'étude en $\mathrm{ml} / \mathrm{hr}$, commençant en période préopératoire, sont illustrées à la Figure 1 et à la Tableau II. En général on observe une chute de la diurèse le jour de l'opération, jour 1 , qui tend à disparaître par la suite. On remarque que la diurèse d'un sujet se détache nettement de l'ensemble du groupe pour atteindre une valeur se chiffrant à $187 \mathrm{ml} / \mathrm{hr}$ au $4^{\mathrm{e}}$ jour. L'observation de ce malade continuée jusqu'au $7^{\mathrm{e}}$ jour a confirmé le maintien de ce haut débit urinaire.

\section{Osmolalité urinaire (Fig. 2, Tableau III)}

La Figure 2 représente l'osmolalité urinaire mesurée sur chaque collection. Malgré une grande dispersion des données on note que la très grande majorité

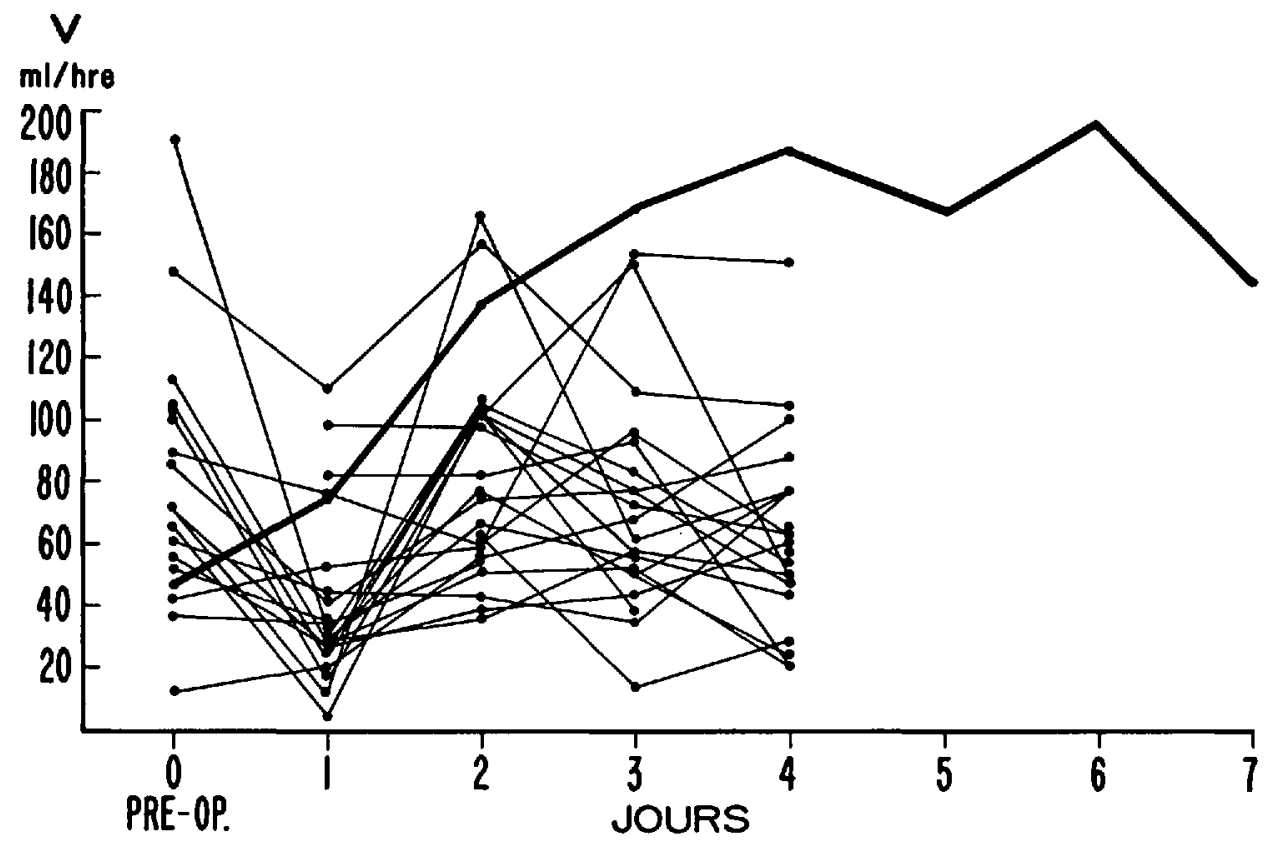

Figure 1. Débit urinaire. 
TABLEAU II

DÉBtTS URINATRES HORAIRES (en ml)

\begin{tabular}{lcccccccc}
\hline \hline Dossier & Preop. & $1^{\mathrm{er}} \mathrm{jr}$ & $2^{\mathrm{e}} \mathrm{jr}$ & $3^{\mathrm{e}} \mathrm{jr}$ & $4^{\mathrm{e}} \mathrm{jr}$ & $5^{\mathrm{e}} \mathrm{jr}$ & $6^{\mathrm{e}} \mathrm{jr}$ & $7^{\mathrm{e}} \mathrm{jr}$ \\
\hline $69-84-682$ & 88.5 & 76.5 & 59.1 & 153 & 150 & & & \\
$69-72-205$ & 113.3 & 27.1 & 76.5 & 50 & 77.5 & & & \\
$69-109-898$ & 70.8 & 27.2 & 38.7 & 43.6 & 60.9 & & & \\
$69-101-090$ & 190 & 31.2 & 105.6 & 84 & 54 & 168 & 195 & 144 \\
$69-110-037 *$ & 46.1 & 75 & 137.5 & 168.7 & 187.5 & 168 & & \\
$69-110-073$ & 148 & 110 & 157 & 109 & 104 & & \\
$69-110-161$ & 100 & 29 & 65.8 & 56 & 44 & & \\
$69-110-242$ & 104 & 18.8 & 104 & 39 & & \\
$69-100-768$ & 70 & 12 & 166.6 & 61.4 & 76.6 & & \\
$69-110-290$ & 105 & 24.1 & 101 & 150 & 50 & & \\
$69-110-489$ & 55.4 & 30 & 36.1 & 57.5 & 48.3 & & \\
$69-110-654$ & & 81.2 & 81 & 93 & 20.8 & & \\
$69-94-879$ & 63.3 & 4.1 & 102.5 & 56.6 & 65 & & \\
$69-110-634$ & & 29.1 & 50.8 & 51.6 & 24 & & \\
$69-110-761$ & 42.5 & 52.5 & 59.4 & 95.8 & 62.5 & & \\
$69-110-749$ & 60.8 & 44 & 42.5 & 35 & 76.4 & & \\
$69-110-847$ & & 98.3 & 97.5 & 72 & 64.1 & & \\
$69-110-831$ & 37.5 & 35 & 54 & & & \\
$69-99-360$ & 51.6 & 35 & 62.5 & 14.1 & 27 & & \\
$69-110-947$ & 70.8 & 27 & 102.4 & 76.8 & 88.3 & & \\
$69-64-506$ & 86.6 & 42 & 75.8 & 77.5 & 47.2 & & \\
$69-110-760$ & 12.3 & 20 & 56 & 67.9 & 100 & \\
\hline
\end{tabular}

*Cas positif de l'étude.

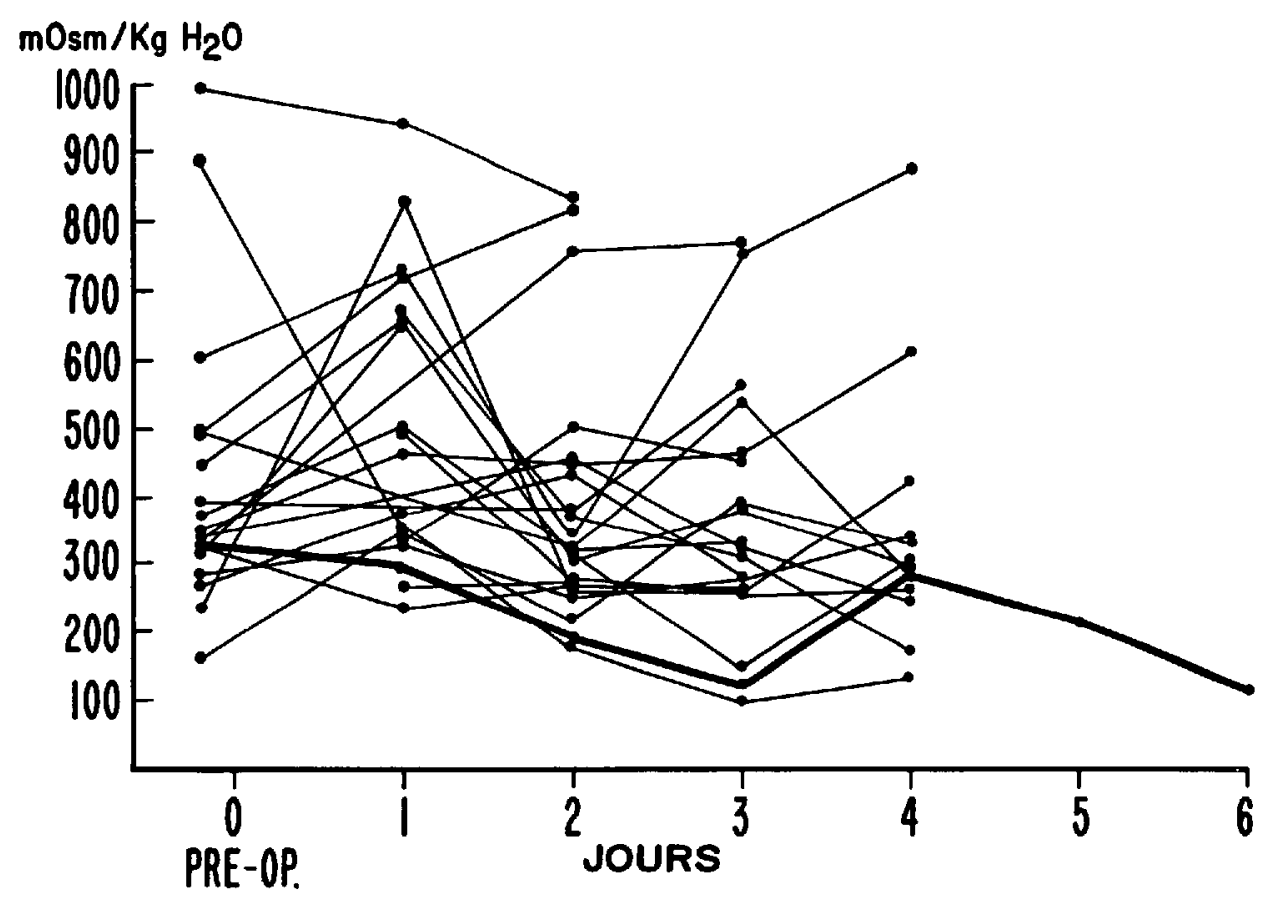

Figure 2. Osmolalité urinaire.

des valeurs se chiffre au-delà de $285 \mathrm{~m}$. osm. par kilo d'eau (Tableau III) démontrant que, dans l'ensemble, les malades excrètent une urine concentrée. A l'inverse de la diurèse (Fig. 1), l’osmolalité urinaire est généralement plus élevée au jour 1. 
Le trait prononcé au bas du groupe indique l'hypotonie persistante de l'urine du cas ayant présenté la polyurie notée précédemment.

TABLEAU III

OsMOlalité URinatre (m. osmol./ $/ \mathrm{kg} \mathrm{H}_{2} \mathrm{O}$ )

\begin{tabular}{|c|c|c|c|c|c|c|c|c|}
\hline Dossier & Préop. & $1^{e r} \mathrm{jr}$ & $2^{\circ} \mathrm{jr}$ & $3^{\mathrm{c}} \mathrm{jr}$ & $4^{e} \mathrm{jr}$ & $5^{\mathrm{c}} \mathrm{jr}$ & $6^{e} \mathrm{jr}$ & $7^{e} \mathrm{jr}$ \\
\hline $69-84-682$ & 226 & 377 & 440 & 271 & & & & \\
\hline $69-72-205$ & 376 & 503 & 311.5 & 334 & & & & \\
\hline $69-109-898$ & 333 & & 759 & 765 & & & & \\
\hline $69-101-090$ & 449 & 651 & 305 & 382 & 293 & & & \\
\hline $69-110-037^{*}$ & 316 & 291 & 181 & 125 & 275 & 212 & 110 & \\
\hline $69-110-073$ & 236 & 824 & 254 & 252 & 263 & & & \\
\hline $69-110-161$ & & 270 & 266 & 270 & & & & \\
\hline $69-110-242$ & 398 & & 382 & 562 & & & & \\
\hline $69-100-768$ & 327 & 239 & 270 & & & & & \\
\hline $69-110-290$ & 314 & 657 & 320 & 147 & 304 & & & \\
\hline $69-110-489$ & 490 & 723 & 815 & & 488 & & & \\
\hline $69-110-654$ & & 338 & 505 & 453 & & & & \\
\hline $69-94-879$ & 343 & & 460 & 322 & 247 & & & \\
\hline $69-110 \cdot 634$ & & 491 & 277 & 258 & 424 & & & \\
\hline $69-110-761$ & 287 & 330 & 250 & 272 & 338 & & & \\
\hline $69-110-749$ & 498 & & 325 & 543 & 275 & & & \\
\hline $69-110-847$ & 165 & 349.5 & 220 & 382 & 331 & & & \\
\hline $69-110-831$ & 978 & 946 & 837 & & & & & \\
\hline $69-99-360$ & 605 & 731 & 343 & 751 & 878 & & & \\
\hline $69-110-947$ & 887 & 356 & 176 & 99 & 135 & & & \\
\hline $69-64-506$ & 350 & 466 & 456 & 465 & 611 & & & \\
\hline $69-110-760$ & & 673 & 386 & 312 & 173 & & & \\
\hline
\end{tabular}

*Cas positif de l'étude.

Cas positif (Fig. 3)

Il s'agit de M. D.T., 50 ans, dossier 69-110-037, qui a subi une arthroplastie par cupule. L'anesthésie au méthoxyflurane a duré 4 hres 40 min. Les pertes sanguines peropératoires ont été évaluées entre 950 et $1000 \mathrm{cc}$. Le malade a reçu en cours d'intervention: glucose 5 pour cent eau, $1500 \mathrm{cc}$; lactate Ringer, $1000 \mathrm{cc}$; $\mathrm{NaCl} 0.9$ pour cent, $300 \mathrm{cc}$; sang entier, $500 \mathrm{cc}$; et globules r. sédim., $300 \mathrm{cc}$ pour un total de $3600 \mathrm{cc}$. Mille cc de glucose 5 pour cent dans l'eau ont complété ses ingesta pour le jour opératoire (jr. 1) soit $4600 \mathrm{cc}$.

Le malade légèrement hémo-concentré à l'examen préopératoire n'avait présenté aucune autre particularité que celle d'un patient obèse. Tous ses examens de laboratoire étaient normaux y compris une urée sanguine à $40 \mathrm{mg}$ pour cent, une créatinine plasmatique à $1.2 \mathrm{mg}$ pour cent et une densité urinaire à 1023 .

Balance hydrique moyenne (Fig. 4)

La Figure 4 illustre la balance hydrique moyenne par 24 heures du groupe des 22 malades étudiés pour la période des 5 jours d'observation en comparaison avec le sujet polyurique.

La valeur faiblement positive du bilan du groupe disparait si l'on estime les pertes insensibles à environ $400 \mathrm{ml} / 24$ heures.

Il en est différemment du sujet D.T. qui, malgré une hydratation moyenne d'audelà de $3000 \mathrm{ml} / \mathrm{jr}$, présente un déficit d'environ $700 \mathrm{ml} / \mathrm{jr}$ sans tenir compte des pertes insensibles. Malheureusement, il fut impossible d'obtenir le poids du malade à cause d'appareil platré et d'immobilisation commandés par la nature de l'intervention. 


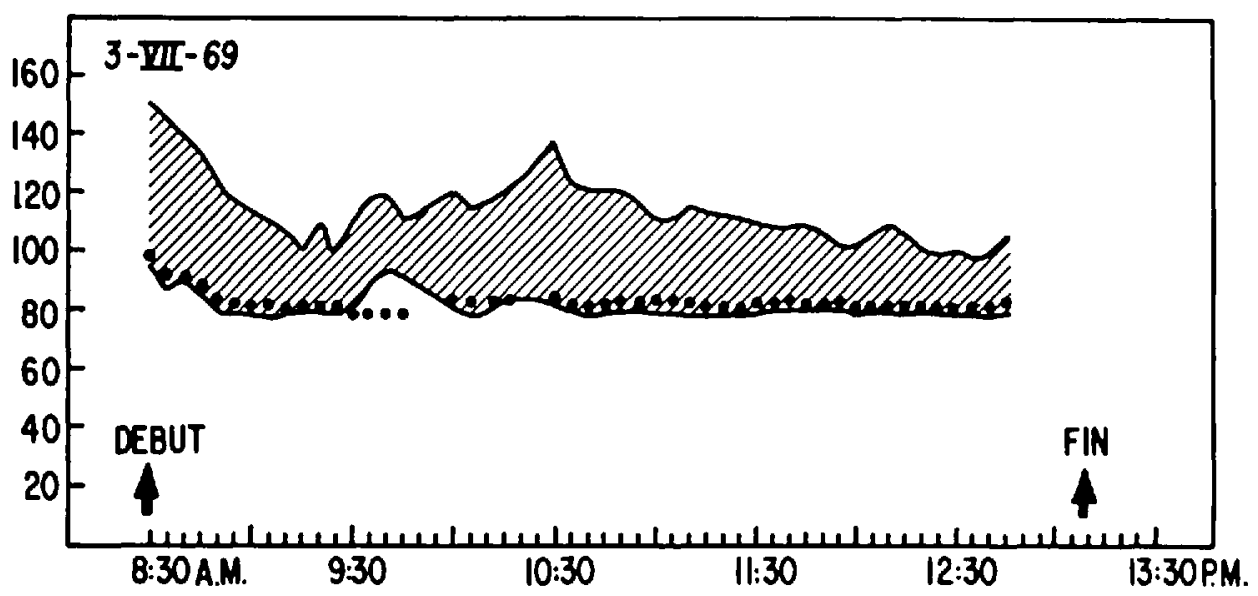

Ficure 3. Cas positif, M. D.T. Diagnostic: ostéoarthrite hanche gauche; état préanesthésique: bon. Intervention: arthroplastie par cupule sous anesthésie au methoxyflurane. Créatinine, $1.1 \mathrm{mg}$ pour cent; TA, 130/90; densité urinaire, 1023; urée, $41 \mathrm{mg}$ pour cent; Hb, 15.6, HT 45.5; obésité modérée; ECG, alterations non specifiques. Agents anesthésiques: Pentothal$\mathrm{N}_{2} \mathrm{O} / \mathrm{O}_{2}$-Penthrane. Technique: i.v.-masque-intubation-sfrp-respirateur Bird Mark Iv/vwr. Pentothal $320+80 \mathrm{mg}$, anectine $100 \mathrm{mg}$, d-tubocurarine $3+27 \mathrm{mg}$, démérol $20+20 \mathrm{mg}$, atropine $0.4 \mathrm{mg}$, prostigmine $0.5 \mathrm{mg}$. Pertes sanguines (peropérative) $950 \mathrm{ml}$.

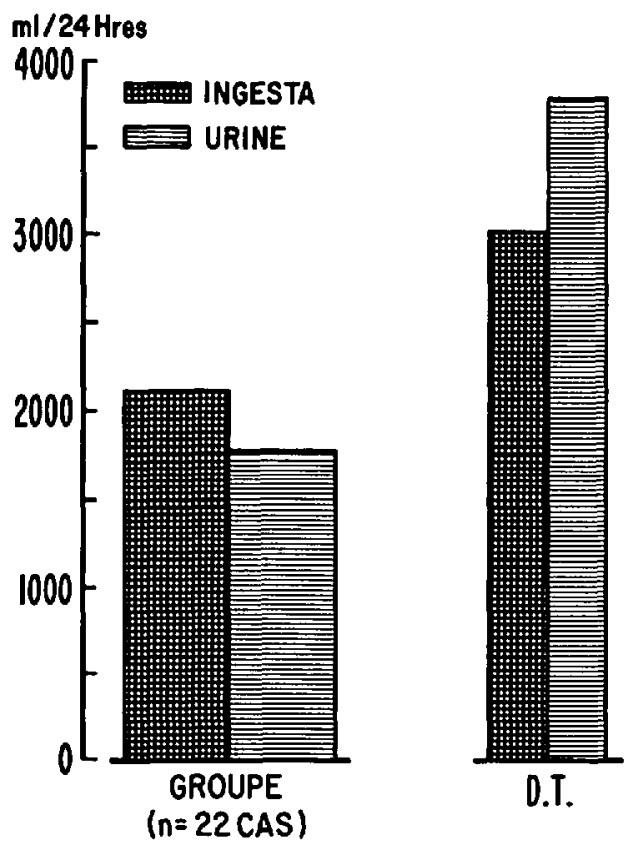

Figure 4. Balance hydrique moyenne des sujets de l'étude en regard de M. D.T., cas positif de l'étude.

Balance hydrique quotidienne de ce même malade (Fig. 5)

La diurèse s'élève jusqu'à $4700 \mathrm{ml}$ le $6^{\mathrm{e}}$ jour. L'aspect fortement positif du bilan du $1^{\text {er }}$ jour s'explique par le remplacement des pertes extra-rénales (hémorragies peropératoire, exsudation, évaporation) non figurées ici. On remarque la forte 


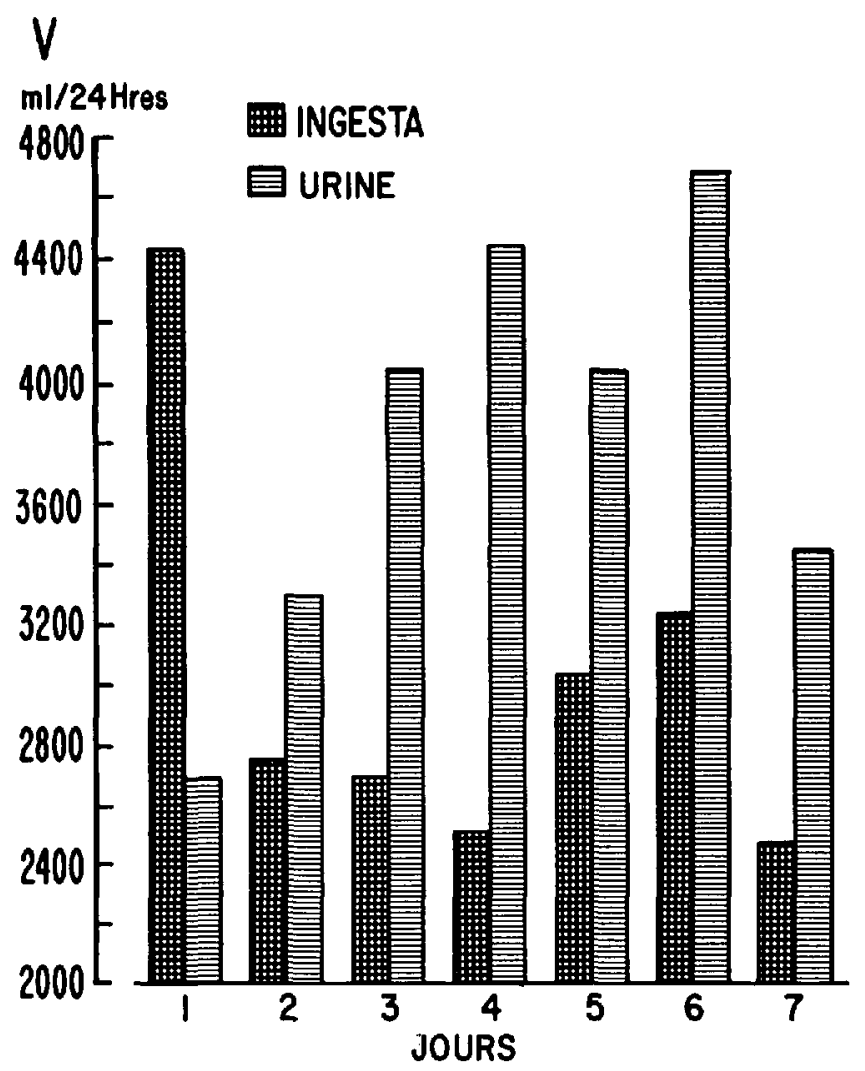

Frgure 5. Balance hydrique quotidienne du cas positif.

diurèse des jours subséquents dépassant de beaucoup l'apport liquidien de chaque jour. Il convient de souligner qu'au cours de cette période d'observation, l'urée sanguine et la créatinine plasmatique sont demeurées identiques aux valeurs de contrôle préanesthésiques qui étaient normales: $40 \mathrm{mg}$ pour cent et $1.1 \mathrm{mg}$ pour cent respectivement.

Soupçonnant à l'avance la nature néphrogénique de ce syndrome polyurique, il nous a semblé hazardeux de soumettre ce malade à une épreuve de déshydratation. Nous avons jugé préférable de quantifier sa capacité de concentration urinaire par une épreuve à la pitressine qui fut pratiquée au $5^{\mathrm{e}}$ jour.

\section{Epreuve à la pitressine (Fig. 6)}

Cette épreuve consiste à mesurer l'effet d'une stimulation maximale du rein par la pitressine au cours de périodes successives de 30 minutes. Les paramètres mesurés ont été la diurèse en $\mathrm{ml} / \mathrm{min}$, l'osmolalité urinaire par rapport à l'osmolalité plasmatique (U/P osmol.) et la mesure de la filtration glomérulaire à l'aide de la clearance de la créatinine. La dose initiale de pitressine aqueuse est de 50 milli-unités intraveineuses puis de 1000 milli-unités/heure soit 16 milli-unités/ minute.

$\mathrm{Au}$ cours de l'épreuve du $5^{\mathrm{e}}$ jour postopératoire on note que la pitressine administrée ne produit pas d'abaissement de la diurèse, que le rapport $U / P$ osmol. 

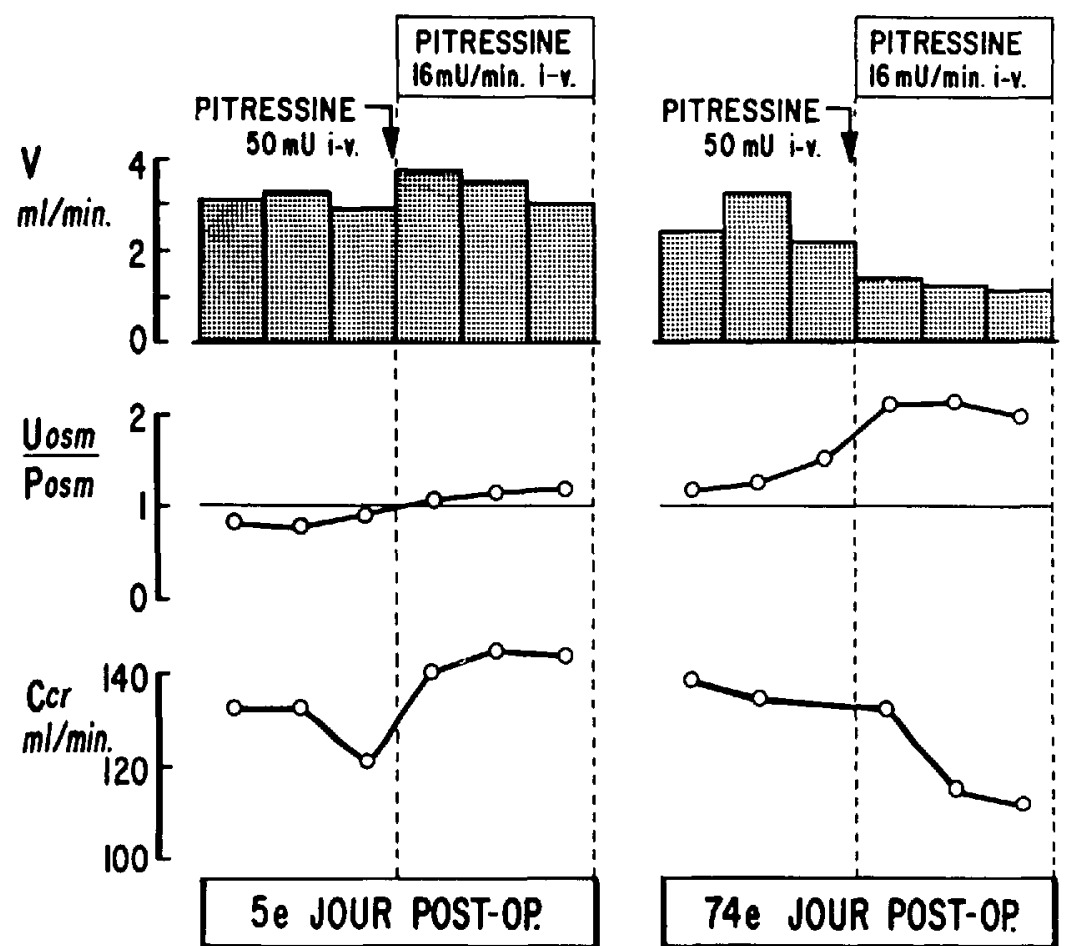

FIgUre 6. Epreuve à la pitressine du cas positif.

dépasse à peine l'unité et que la filtration glomérulaire reste dans des limites toutà-fait normales. Ceci témoigne donc d'un défaut sélectif du pouvoir de concentration du rein en présence d'une fonction glomérulaire normale.

Il nous a été possible de répéter cette même épreuve 69 jours plus tard et il est intéressant de constater que le malade répond à la pitressine par un abaissement de la diurèse-minute d'environ 50 pour cent et par l'élévation de son osmolalité urinaire à 2.1 fois celle du plasma. La clearance de la créatinine est demeurée normale. Il s'agit donc d'une amélioration sensible du pouvoir de concentration qui cependant demeur encore inférieure à la normale.

\section{Discussion}

Nos observations indiquent l'occurrence d'un cas de syndrôme polyurique résistant à la pitressine en l'absence d'insuffisance rénale sur un total de 22 anesthésies au méthoxyflurane.

Le nombre de cas étudiés ne nous permet évidemment pas d'analyse statistique. Il nous semble cependant que l'incidence de 17 pour cent rapportée par Crandell ${ }^{2}$ en 1966 est nettement supérieure à ce que suggèrent nos données.

Il est permis de croire que le mode d'utilisation et la dose administrée de méthoxyflurane pourraient être reliés au degré de néphrotoxicité de cet agent. Selon notre expérience l'utilisation du Penthrane ${ }^{\circledR}$ à dose strictement analgésique par "Pentec" résulte en l'administration de quantité beaucoup plus faible que celle réalisée lors d'anesthésie au Penthrane comme agent principal et administré par 
le vaporisateur Ohio No 8 tel qu'utilisé dans la majeure partie des cas de Crandell.

Il n'en reste pas moins que le méthoxyflurane demeure potentiellement néphrotoxique. La nature précise de cette néphrotoxicité reste à élucider. La possibilité d'une origine neuro-hypophysaire du défaut de concentration de l'eau est éliminée par l'absence de réponse du rein à la vasopressine exogène.

Toutefois, plusieurs cas mentionnés dans la littérature ont développé une rétention azotée au cours de l'épisode polyurique. ${ }^{3,4}$ Ceci suggère donc la possibilité d'une ischémie rénale aigue ou une toxicité directe sur les mécanismes de transport tubulaire sousjacents au processus de concentration de l'urine.

La composante ischémique serait en accord avec le mode d'administration et la dose si l'on s'en rapporte aux effets hémo-dynamiques du méthoxyflurane si bien décrits par Hudon, Jacques, Déry et coll..$^{5}$ en 1963.

Faisant suite à la phase hyperkinétique de l'analgésie ou de l'anesthésie légère, le méthoxyflurane en surdosage produit une dépression cardiaque marquée que compense une vaso-constriction périphérique proportionnelle, suffisante au maintien de la tension artérielle. La réaction vasculaire est si intense qu'elle produit la pâleur marquée des téguments, la disparition du pouls capillaire, une cyanose sub-unguéale et un éveil prolongé - syndrome bien connu de l'anesthésie profonde au méthoxyflurane. L'hypotension signe un surdosage qui dépasse le mécanisme de compensation.

Dans l'optique de ces données, si l'on revient au malade positif de l'étude $\mathrm{M}$. D.T. (voir Fig. 3) la tendance à l'hypotension per anesthésique (TA préopératoire 130-140 syst.) laisserait supposer une anesthésie plus profonde que prévue, la vasoconstriction réactionnelle s'ajoutant à celle de l'hémorragie peropératoire.

Quels qu'en soient les mécanismes sousjacents, les observations de l'étude imposent une réserve dans l'utilisation de cet agent en particulier chez les malades qui présentent au préalable une réduction fonctionnelle rénale.

\section{RÉSUMÉ ET CONCLUSION}

Nous avons étudié la fonction rénale de 22 malades anesthésiés au méthoxyflurane. Un cas a présenté une polyurie marquée avec persistance d'une excrétion d'urine hypotonique et un abaissement du pouvoir de concentration en réponse à la vasopressine.

Certains facteurs étiologiques ont été brièvement discutés ainsi que les mécanismes physio-pathologiques possibles.

Le syndrome polyurique décrit et son incidence imposent une surveillance de tous les malades quant à l'équilibre liquidien, la natrémie, l'azotémie et une réserve dans l'emploi du méthoxyflurane en présence d'atteinte rénale.

\section{SumMary}

Renal investigation was done on 22 patients anaesthetized with methoxyflurane. One patient developed marked diuresis, persistent low osmolality, and a lessened urinary concentrating power in response to vasopressin. 
Some etiological factors along with a possible physiopathological mechanism have been briefly discussed.

The high incidence of this polyuric syndrome calls for a close observation of the urine output of all patients subjected to methoxyflurane anaesthesia. This agent would appear contraindicated in patients with reduced renal function.

\section{REMERCIEMENTS}

L'étude de la fonction rénale de ces malades s'est faite avec le précieux concours du docteur Julien Marc-Aurèle de la section de Néphrologie de l'HôtelDieu de Montréal.

\section{BIBLIOGRAPHIE}

1. Lebowitz, M. H. Nephrogenic Diabetes Insipidus Following Methoxyflurane Anesthesia: A Report of Two Cases. Anesth. \& Anal. 48: 233 (1969).

2. Crandell, N. V.; Pappas, S. S.; \& Macdonald, A. Nephrotoxicity Associated with Methoxyflurane Anesthesia. Anesthesiology, 27: 591 (1966).

3. PadDock, R. B.; Parker, J. W.; \& Suadahny, N. P. The Effects of Methoxyllurane on Renal Function. Anesthesiology. 25: 707 (1964).

4. Austin, W. H. \& WrLANDRX, J. P. Methoxyflurane and Renal Function. Correspondence. Anesthesiology. 28: 637 (1967).

5. Hudon, F.; Jacques, A.; DÉRY, R,; Roux, J.; \& MÉnaRd, J. Effets Respiratoires et Hémodynamiques de l'Anesthésie au Methoxyflurane. J. Soc. Canad. Anesth. 10: 460 (1963). 